Physiotherapy Supervision of Home Trainers in Interprofessional Reablement Teams

\begin{abstract}
Physiotherapists (PTs) in reablement are responsible for the supervision of support personnel, referred to as home trainers (HTs), who carry out training and initiatives. There is a lack of knowledge about the significance of physiotherapy supervision in reablement. The aim of this study was to explore the content of PTs' supervision of HTs in reablement teams. We conducted fieldwork in seven reablement teams in Norwegian municipalities. The methods included observations of practice and individual in-depth interviews with PTs and HTs. We analysed data thematically through an iterative inductive-deductive process. The results were generated in a social constructionist perspective and situated learning theory guided the analyses. Analyses revealed that supervision included elements of instruction, demonstration and reflection. However, practices varied widely across different teams, especially regarding the reflection aspect, which was highlighted as essential for learning. Frequent meetings, both formal and informal, were essential to enable learning through reflection. This paper identifies and discusses fundamental elements of PTs' supervision practice in reablement teams, which is also relevant for similar interprofessional settings. Managers of reablement programs should be aware of the powerful impact that organizational conditions have on the practice of supervision.
\end{abstract}

Keywords: Interprofessional teamwork, interprofessional learning, situated learning theory, organizational frames, interview, observation 


\section{Physiotherapy Supervision of Home Trainers in Interprofessional Reablement Teams}

Increasing costs of health care services and an aging population are leading to a shortage of health care personnel. As a result of these driving forces, authorities in countries worldwide are reorganizing and changing the priorities of health care services (World Health Organization, 2006). Task shifting (or task sharing) is described as the delegation of tasks to existing or new candidates with narrowly tailored training; it is a potential strategy with which to address challenges stemming from the shortage of human resources in health care services (Fulton et al., 2011; Kakuma et al., 2011). Authorities in several high-income countries have suggested reablement as a resource-utilizing approach to meeting current workforce challenges (Cochrane et al., 2016; Legg, Gladman, Drummond, \& Davidson, 2016).

Reablement is a relatively new interprofessional, team-based approach to homerehabilitation that aims to enable people with - or at risk of - functional decline to cope with everyday life. The service is intensive, time-limited and person-centred, as it is based on the user’s goals (Cochrane et al., 2016). In contrast to traditional rehabilitation, interventions in reablement are, to a large extent, delivered by support personnel without a professional background, often referred to as home trainers (HTs).

The content and organization of reablement services vary across different countries (Legg et al., 2016; Pettersson \& Iwarsson, 2017). However, literature often notes that health care professionals such as physiotherapists (PTs), occupational therapists (OTs) and nurses play an essential supervisory role in reablement (Birkeland, Tuntland, Førland, Jakobsen, \& Langeland, 2017; Fürst \& Høverstad ANS, 2014; Ness et al., 2012; Rabiee \& Glendinning, 2011; Tessier, Beaulieu, McGinn, \& Latulippe, 2016).

PTs, in particular, have knowledge and skills pertaining to bodily structures, body functions and movement, all of which should be assessed and targeted in reablement interventions (Ness et al., 2012). Physiotherapy is concerned with quality and accountability 
(French \& Dowds, 2008), as PTs are specialists in analysing, promoting and supporting human movement (Nicholls \& Gibson, 2010). In their work, PTs integrate theoretical knowledge, clinical experience and relational, ethical and contextualized skills (Dahlgren, Richardson, \& Kalman, 2004). However, knowledge of the complex and multifaceted body is more or less interwoven in physiotherapy practice and is not easy to describe verbally (Nicholls \& Gibson, 2010). Normann, Sørgaard, Salvesen, and Moe (2014) argue that practical knowledge in physiotherapy is available only through first-hand experience and that such knowledge is achieved through reflection during and following action, in accordance with the concepts of 'reflection-in-action' and 'reflection-on-action' (Schön, 1991).

Studies describe PTs’ (serving as practice educators) supervision of PT students (Fogstad \& Christiansen, 2011; Lähteenmäki, 2005; Stainsby \& Bannigan, 2012), specialist PTs' supervision of community PTs (Normann et al., 2014), and paediatric PTs’ supervision of the caregivers of disabled children (Sørvoll, Obstfelder, Normann, \& Øberg, 2016). These studies emphasize that physiotherapy supervision is a process where knowledge is transferred through interaction and reflection and that action, context and culture are essential for learning (Korpi, Peltokallio, \& Piirainen, 2014). However, literature on PTs’ supervision of non-professionals is sparse, and the content of supervision in reablement services is, to the best of our knowledge, not described.

Supervision of HTs in reablement largely represents the foundation of the service provided to reablement users. The aim of this study was to investigate the content of physiotherapy supervision and knowledge transfer in reablement teams. Study results may contribute to improving both the knowledge and skills applied in the provision of the service.

\section{Theoretical Framework}

Physiotherapy practice is concerned with continuous clinical reasoning, involving various aspects of knowledge and skills. Awareness of body position, quality of movement 
and communication is essential for physiotherapy practice. According to Grimen (2008), these aspects of knowledge are difficult to verbalize and cannot be fully transferred through descriptions.

We have used theory of learning as a socio-cultural process to analyse and understand the transfer of knowledge in reablement. Socio-cultural learning theories conceptualize learning as a complex process of human interaction. From this perspective, learning is considered to be constructed through interaction with others within a certain context; such contexts are referred to as ‘Communities of Practice’ (Wenger, 1998). In defining learning, Wenger (1998) described three hallmarks of communities of practice: First, participants are mutually involved and brought together as a social unit. Second, the community is linked to a joint activity. Third, the participants possess a common repertoire of practices, tools and symbols. This perspective was used as a frame to develop the research question, create the observation- and interview guide and to analyse the data.

\section{Methods}

Aiming to explore the actual interactions in practice, the results were generated based on a social constructionist perspective, which acknowledges the experiences of individuals through their interactions with others as well as through historical and cultural norms (Guba \& Lincoln, 1989).

\section{Study Design}

This is a qualitative, explorative study based on fieldwork in reablement teams. We collected data through a) observations of interactions between PTs and HTs in their work environment; 2) videotaped observations of user interventions; and 3) in-depth interviews with both PTs and HTs based on the observation sessions.

\section{Study Setting}


We obtained data from seven reablement teams within seven different Norwegian municipalities. The municipalities were strategically chosen to obtain a varied population size. Observations of practice were carried out at the participants' workplaces. Observations of supervision and reablement sessions took place in the users' homes. All interviews took place in meeting rooms at the PTs' and HTs' work places.

\section{Participants and Recruitment}

Two members (a PT and a HT) from each of the seven reablement teams were included as participants $(\mathrm{N}=14)$. However, reablement users and other collaborating team members contributed to production of data through their interactions.

Collaborative co-workers in one of the reablement teams did not consent to participate, and the work environment was not observed in this team. However, results from interviews of the PTs and HTs gave rich information about the work environment and collaborative practice in this team as well, and we therefore decided to include these data.

Inclusion criteria for the PT and HT were at least 6 months of experience with reablement. The inclusion criteria for the users were that they were about to start with - or were in the first week of - reablement. All participants had to be able to consent to participation, and users with cognitive impairment were therefore excluded.

General managers of the teams were contacted, and they consented to distribute information about the study to PTs and HTs. The PTs distributed information and recruited users. When a user agreed to participate in the study, the PT contacted the first author (ME) to arrange for data collection.

\section{Data Collection}

The first author collected data between January and June 2016. We visited the triads during the users' first week of reablement, followed by a second visit two to three weeks later. In this study, the key points of the observation guide that was used in the patient-intervention 
observations were 1) treatment setting, 2) content of actions regarding assessments or training, 3) instructions to users, 4) instructions to HTs, and 5) verbal- and non-verbal communication. The intervention was video-recorded to ensure that the situated and interactional accomplishment of practical action were captured (Heath, Hindmarsh, \& Luff, 2011). We primarily remained in the background in order to avoid disturbing the interaction of the participants and used a discreet, handheld camera with a wide video angle to capture all participants.

During the first visit, we observed the user interventions when both the PT and HT were present with the user. Immediately after the first observation, we conducted an interview with the PT focusing on the previous observation and on strategies for supervision.

The second visit included video-recorded observation of the encounter between the HT and user, followed by an interview with the HT. The interview focused on the preceding observation and the HT's experiences with supervision. Interviews with both the PTs and HTs were semi-structured, with open-ended questions and relevant follow-up questions. The interviewees were encouraged to talk freely about their experience with supervision and clinical discussions (Kvale \& Brinkmann, 2009). All interviews were audio-recorded.

In addition to the observations and interviews, field observations of the reablement teams' work environment were conducted to analyse the context that serves as a framework for the practice. The first author followed the PT and HT from six of the reablement teams at their work place. Observations in the office, lunchroom and meeting rooms captured both formal and informal meetings and interactions between the team members.

\section{Data Analysis}

A thematic analysis was conducted through an inductive-deductive process, where the inductive analysis was used to allow the patterns, themes and categories of the data material 
to emerge (Lincoln \& Guba, 1985), and the deductive theoretical interpretation contributed to developing and validating the findings (Creswell \& Poth, 2017).

The interviews were transcribed verbatim, while the video observations were formatted as text through descriptions in a scheme where six aspects of interactions were categorized: 1) what is done? 2) how is it done? 3) explanation or reasoning, 4) instructions provided, 5) guidance provided, and 6) interaction. The descriptions included both verbal talking and bodily interactions. The cataloguing of video-recorded data was inspired by Heath, Luff, and Sanchez Svensson (2007) and aimed to capture the multiple aspects of interaction in video observations.

Further, we identified and coded meaningful units in all text material. During this process, all data materials were linked together, supplementing each other, through common code groups and themes. The interviews elaborated the observations through the insights of both the PTs' and the HTs' experiences with physiotherapy supervision. Congruent data from all three methods supported and validated each other, while discrepancies were further analysed to achieve an understanding of inconsistencies. Simultaneously, the three authors engaged in theoretical reading and discussions. The analyses were performed as an iterative process, continuously moving between an inductive and a deductive perspective and between the data material and relevant theory. The triangulation, reflexivity and theoretical interpretation contributed to the validation of the findings.

The analysis was supported by the use of QSR NVivo 11 (QSR International), a qualitative data analysis software.

\section{Ethical Considerations}

This study was approved by the Norwegian Center for Research Data (Ref number 44747) and was conducted according to the Helsinki Declaration (World Medical 
Association, 2017). All participants received written information about the study and provided written consent before the data production.

\section{Results}

Three main themes emerged through the analysis: 1) Forms of supervision; instruction, demonstration and reflection, 2) working together, and 3) home trainers’ engagement. Citations and excerpts from the observations are presented consecutively.

\section{Instruction, Demonstration and Reflection}

Three different forms of supervision were employed during the observations. The first form;instruction revolved particularly around what to do. This was often defined in a reablement plan, which was based on the PT's (or the PT in collaboration with other team members') assessments and the user's goals; it usually included exercises or daily activities that the user was supposed to do. These instructions were often transferred through written information, in the form of a rehabilitation plan or an exercise map that included descriptions and drawings of the exercises. In the team where the HT was a member of the home care service and not co-located with the therapists, the HT highlighted that this written information was a central source of supervision. The HT explained that she had not felt the need to contact the PT for any further supervision:

The PT sends me a, what should I call it... a description. The exercises are carefully described as well as the user's goals. Everything you need to know is there, you know, for the entire period. 'The startup date is... the user’s goals are... perhaps to increase strength and balance', Well, we just have to carry it out (HT).

In addition to the instructions, the second form of supervision referred to demonstration of how to perform the intervention. In contrast, the other HTs claimed that a drawing of the exercises was not always sufficient and that it was essential for the PT to demonstrate how the exercises should be performed. They also emphasized the importance of 
practicing the exercises themselves, which enabled them to perceive and experience how different positions targeted different muscles. Supervision where the PT demonstrated the exercises often took place in the user's home but could also happen in the office, without the user present.

PTs that visited the user together with the HT were able to carry out supervision related to the user's individual technique and quality of movement. One PT said:

For example, the lady we visited earlier today, who was doing her hip abduction exercise beside the kitchen counter. [The purpose is] to stabilize, not just 'topple over', you know. They need to understand how the user should be moving to target the right muscles. There are several ways to compensate. Consequently, the user will not achieve the effect that I had planned for. That's why it’s important to demonstrate and further explain the importance of doing the exercise right. (PT).

The third form of supervision concerned reflection about why initiatives were done. Through fieldwork, we observed that the PTs justified their choice of exercises and techniques based on the user's goals and based on the constraints of the user's physical abilities. They emphasized that the HTs achieved an understanding of the clinical reasoning in order to tailor the interventions to the individual user. Reflecting together enabled a common understanding and shared knowledge. As one PT explained:

You know, it's important that they [the HTs] understand and observe the same as I do. Sometimes we practice here in the office. We carry out the exercises to experience how it should be done; 'what happens if you get a little support here?' and 'can you feel this and that?'[...] I emphasize that they should know how it feels, and learn what to look for' (PT). 
Instruction and demonstration were seen in all the observed teams. However, reflection, which enabled a deeper understanding among the HTs was more frequent in reablement teams in which the PT and HT met frequently during the workday. This is presented in the following section.

\section{Working Together}

All of the reablement teams had established formal meetings where all team members gathered together. The frequency of these meetings varied across teams, from every second week to once a day. The participants discussed both organizational and coordination issues, as well as issues regarding reablement users - including user goals, physical capacities and constraints. In addition to the scheduled meetings, some of the teams had arranged courses where the PT demonstrated exercises based on requests from the HTs.

Even though all the teams arranged formal meetings, they argued that informal meetings and the day-to-day communication were at least as important. With the exception of one team in which the HTs were organized under the home care services, the team members were located together. Observations and interviews revealed that all the team members were communicating at the office, during lunchtime, through phone calls and in the car between the users' homes and the office. The discussions involved the user's goal, physical abilities and exercises. They also discussed their own performance during the user visit in order to evaluate how both the PT and the HT could adjust and improve the approach. The HTs reported a low threshold for contacting the PTs if they were unsure of something. Co-located PTs and HTs said that the common premises were essential to achieving close collaboration. One PT explained that the daily contact enabled less-hierarchical communication by creating dialogue and discussion rather than a session where the PT lectured the HT:

We don’t have anything that we call supervision-sessions specifically. It all happens through day-to-day communication at the office. [...] If we had called it supervision, I 
think it would have been more of a "top-down" approach, in that the PT always was the one with the right answer. I don’t think that would have been advantageous for the work environment. The informal contact and low threshold to discuss with each other is better (PT).

Although much of the supervision happened in the office, it was explained that the most important arena for supervision was the user's home when both the PT and the HT were present. Usually, the PT and HT visited the user together during the first days of reablement to conduct assessments and implement exercises. This was highlighted as essential, as one PT noted:

Because it happens a lot during the user meetings, that is challenging to write or explain if they [the HTs] are not present [...] so it is important that the HT is present in these meetings (PT).

The participants highlighted the benefits of visiting the user together as often as possible. Most of the HTs reported that it was a common practice to ask the PT to attend training sessions throughout the reablement period if they felt uncertain. Occasionally, the PT would attend the training session, even though there was no particular need, simply to follow up on the user's progress and to provide feedback on the HT's interaction with the user.

\section{HTs’ Motivation and Involvement}

The PTs claimed that they often had to supervise the HT indirectly through the communication with the user because the supervision was, to a large extent, conducted in the users' homes. The PTs expected the HTs to absorb the information that was addressed to the users, which required the HTs to be highly observant:

I have to learn from the PT and the user's communication, so you have to be observant. You have to be ‘all in’ even though you're just an observer. You can’t just sit around. You have to watch and listen carefully (HT). 
The PTs also highlighted the HTs’ motivation and engagement as essential to the supervision, as one said:

She is a quick learner and she is genuinely interested in her tasks. She reads a lot and is truly passionate about the work she is doing. My experience is that it is very easy to transfer knowledge to her because she is interested and asks questions if she is unsure (PT).

The HTs reported that they were more motivated when they received new challenges and that they positively perceived reflections and discussions with other team members.

\section{Discussion}

The results show that different forms of supervision, namely instruction, demonstration and reflection, are essential to transferring professional knowledge from PTs to HTs. Shared reflections were emphasized as especially crucial to the learning process. Formal and informal meetings, as well as the HTs' motivation and engagement, particularly enabled reflections.

\section{Practical Knowledge}

Mostly, PTs distributed reablement plans and training programs as written information to the HT. However, almost all the participants reported that both demonstration of exercises and observations of PTs’ interactions with users were essential. Reablement approaches involve practical actions that are complex and contextually unique. Such approaches require knowledge about how to move in a functional and efficient way, how to support and guide the user through verbal and bodily instructions and, at the same time, observe and assess for compensating movements. Verbal instructions are not always sufficient to transfer contextspecific practical knowledge. Donald Schön wrote:

Often we cannot say what it is we know. When we try to describe it we find ourselves at a loss, or we produce descriptions that are obviously inappropriate. Our knowing is 
ordinarily tacit, implicit in our patterns of action and in our feel for the stuff with which we are dealing. It seems right to say that our knowing is in our action (Schön, 1983, p. 49)

This is consistent with educational theories of practice, which emphasize active learning techniques and claim that learning is not solely transmitted by direct instruction (Biggs, 1999).

The example mentioned earlier, where the HT was satisfied with the written information about exercises, is an example of what Schön (1991) referred to as a technical approach, which he critiqued as a potential danger for practice. The HT considered her task to be carrying out the content of the written instruction, without any further adjustments to the context of the intervention. In a qualitative study of reablement services, Rabiee and Glendinning (2011) also found that some HTs considered their job as passively 'standing and watching', without any interference due to adjustments. Although our study revealed that reflection in some reablement teams was sparse, other teams with closer collaboration and frequent meetings emphasized that reflection and discussion were essential for the HTs' learning. An ongoing study is exploring the content and quality of service delivered in reablement teams that employ varied supervision practices (Eliassen, Henriksen, \& Moe, in review).

PTs in this study reported that the discussions enabled the HTs to understand the users' situation better, which further enabled them to take clinical decisions based on their acquired knowledge. Reflective practice is known to be essential for learning practical skills in health and social care services (Kinsella, 2010), as well as in physiotherapy (Ernstzen, Bitzer, \& Grimmer-Somers, 2010). By visiting the users together, the PT and HT created 'reflection-in-action', which Schön (1991) described as reflection while acting. Both informal and formal meetings outside the user's home enabled consecutive 'reflection-on-action' 
(Schön, 1991). Frequent meetings and shared practice were important prerequisites for the discussions.

\section{Learning in a Professional and Collaborative Environment}

Analysis revealed that reablement teams that engaged in common meetings and close collaboration had more thorough discussions of professional issues compared with teams where the PT and HT rarely met each other. Birkeland et al. (2017) also found that learning in reablement depended on how closely the participants collaborated. They indicated that organizing reablement teams outside the home care service could contribute to greater interdisciplinary collaboration compared with integrated teams within the traditional home care services. In accordance with social theories, learning is situated (Lave \& Wenger, 1991) and is largely dependent on our experiences of participation in daily life, particularly when learning entails engaging in 'communities of practice' as described by Wenger (1998). The reablement teams investigated in this study may be seen as a type of community of practice.

Participants in this study noted that close collaboration and informal meetings produce a non-hierarchical environment. This complies with a central hallmark of Wenger's definition of community of practice, namely a social unit characterized by joint enterprise (Wenger, 1998). A study that explored reablement employees’ experiences argued that regular meetings and a common arena for communication enabled the therapists to appreciate the HTs' work (Hjelle, Skutle, Førland, \& Alvsvåg, 2016). Results from our study revealed that informal meetings and day-to-day communication was the most important arena for supervision. This required flexible working where PTs could attend HTs user-encounters whenever it was needed. Other studies have also reported that a flexible structure in interprofessional teams is important (Randsström, Wengler, Asplund \& Svedlund, 2012; Eliassen, Henriksen \& Moe, 2018). 
The participants reported that the HTs' engagement was essential to the transfer of knowledge. The PTs' supervision in the context of a user visit appeared to be challenging due to the ambiguous demand of interacting with the user and at the same time supervising the HT. The PT often conducted supervision indirectly through the communication with the user, which required an observant and engaged HT. It has been reported that the HTs' motivation is necessary in reablement (Rabiee \& Glendinning, 2011). According to Wenger (1998), active participation is an important aspect of learning in a community of practice.

In teams where PTs and HTs worked closely together, the PTs were present during the users' home visits on several occasions. Both the PT and the HT could engage mutually in professional discussions based on their common knowledge about the user. Moe and Brataas (2016) reported that reablement approaches require several types of competences, and thereby several professions should be involved in patient assessment and initiatives. A pluralistic approach is valued, as it provides a range of interpretations and views due to the complexity of care (Reeves, Lewin, Espin, \& Zwarenstein, 2010).

\section{Teamwork or Collaborative Work}

Joint discussions produced common knowledge and understanding of the user's situation; such knowledge may be seen as a shared repertoire which, according to Wenger (1998), is considered to be essential for a learning environment. However, this was not observed in the team where the PT and HT were not co-located and had few meetings. Ultimately, we question the use of the concept 'team' in these cases. According to Reeves et al. (2010), there are different forms of interprofessional work. 'Teamwork' is characterized by team identity, interdependence between team members and shared responsibility, in line with the observations of the co-located and highly reflective teams. However, the reablement teams with limited reflective supervision were more comparable with what Reeves et al. (2010) define as ‘collaboration', where the shared identity and integration of individuals are less 
important. Birkeland et al. (2017) also found that reablement team members that experienced close collaboration appeared to have an effective qualitative and quantitative follow up of the users. This has also been reported in team-work in similar health care settings (Thylefors, Persson, Hellström, 2005).

Although we have described contrasting findings regarding supervision in reablement due to collaboration, it is not our intention to point out 'good' or 'bad' reablement teams. According to Reeves, Xyrichis, and Zwarenstein (2018), the design of the team needs to match the clinical purposes. The service's intention, including the definition of the target population, must guide the choice of design. Birkeland et al. (2017) argue that the organization of reablement services is a question of resources, which also favour local adaptations. However, we argue that complex interventions in reablement services - with a demand for specialized and individually tailored approaches - may benefit from the learning processes used in the more collaborative interprofessional teams, which emphasize reflection in supervision.

\section{Study limitations}

Our results stem from a limited number of participants and teams, and other ways of conducting physiotherapy supervision in reablement may exist. However, the results of this study are relevant to reablement services and to other interprofessional work in similar settings regarding analytical and theoretical generalizations. One of the reablement teams did not consent to perform fieldwork in their work environment, and observations of collaboration between other team members in this team were not performed. This may have limited the ability to contextualize the findings from this particular team. However, we considered data from the observations and interviews as sufficient to conduct relevant analyses. In our study, PTs and HTs were the main subjects, while other team members contributed through discussions and interactions in the interprofessional work environment during fieldwork. 
However, collaboration - including other professions - should be further investigated. This study revealed that reablement practices vary according to service organization and supervision, but their effectiveness across the varied practices has not been studied in this work, and there is a need for further investigation.

\section{Concluding Comments}

This study provides a perspective on knowledge development as a social process, constructed in communities of practice exemplified in the reablement context. The findings of this study show that physiotherapy supervision in reablement involves elements of instruction, demonstration and reflection and that reflection was vitally important to the learning process of the HTs. However, organizational frames such as frequent meetings and close collaboration were essential to promote reflections in and on practice. Nevertheless, the structure of reablement teams needs to be designed in accordance with the clinical purpose and local context of the service.

Task shifting due to reorganization of health care services has resulted in new tasks for professionals as well as for support personnel (Allen, 2014). The content of these tasks must be identified and manifested as part of the practice. This paper contributes to highlighting supervision as an important and potentially resource demanding task that must not be taken for granted in collaborative interprofessional teamwork.

\section{Declaration of Interest}

The authors report no conflict of interest. The authors alone are responsible for the content and writing of the article.

\section{Acknowledgements}


We extend our gratitude to the study participants: physiotherapists, home trainers, users and other team members involved in the research. We would also like to thank The Norwegian Fund for post-graduate training in physiotherapy for funding the study (Grant-id 62716). 


\section{References}

Allen, D. (2014). Re-conceptualising holism in the contemporary nursing mandate: From individual to organisational relationships. Social Science \& Medicine, 119, 131-138

Biggs, J. (1999). What the student does: Teaching for enhanced learning. Higher Education Research \& Development, 18, 57-75

Birkeland, A., Tuntland, H., Førland, O., Jakobsen, F. F., \& Langeland, E. (2017). Interdisciplinary collaboration in reablement-a qualitative study. Journal of Multidisciplinary Healthcare, 10, 195-203

Cochrane, A., Furlong, M., McGilloway, S., Molloy, D. W., Stevenson, M., \& Donnelly, M. (2016). Time-limited home-care reablement services for maintaining and improving the functional independence of older adults. Cochrane Database of Systematic Reviews, 2016, 1-60. doi:10.1002/14651858.CD010825.pub2

Creswell, J. W., \& Poth, C. N. (2017). Qualitative inquiry and research design: Choosing among five approaches. London, UK: SAGE.

Dahlgren, M. A., Richardson, B., \& Kalman, H. (2004). Redifining the reflective practitioner. In J. Higgs, B. Richardson, \& M. A. Dahlgren (Eds.), Developing practice knowledge for health professionals (pp. 15-33): Butterworth Heinemann.

Eliassen, M., Henriksen N., Moe, S. (2018). Variations in physiotherapy practices across reablement settings. Physiotherapy theory and practice.

doi:10.1080/09593985.2018.1481162

Eliassen, M., Henriksen, N., \& Moe, S. The Practice of Support Personnel, supervised by Physiotherapists, in Norwegian Reablement Services (in review). UiT The Arctic University of Norway 
Ernstzen, D., Bitzer, E., \& Grimmer-Somers, K. (2010). Physiotherapy students’ and clinical teachers’ perspectives on best clinical teaching and learning practices: A qualitative study. South African Journal of Physiotherapy, 66, 25-31

Fogstad, L., \& Christiansen, B. (2011). Moving the boundaries: Peer learning between nursing and physiotherapy students. Nordic Journal of Nursing Research, 31, 25-29

French, H., \& Dowds, J. (2008). An overview of continuing professional development in physiotherapy. Physiotherapy, 94, 190-197

Fulton, B. D., Scheffler, R. M., Sparkes, S. P., Auh, E. Y., Vujicic, M., \& Soucat, A. (2011). Health workforce skill mix and task shifting in low income countries: a review of recent evidence. Human Resources for Health, 9, 1. doi:10.1186/1478-4491-9-1

Fürst \& Høverstad ANS. (2014). Fra passiv mottaker til aktiv deltaker [from passive recipient to active participant].

Grimen, H. (2008). Profesjon og Kunnskap [Profession and knowledge]. In A. Molander \& L. I. Terum (Eds.), Profesjonsstudier [Profession studies] (pp. 71-85S). Oslo: Universitetsforlaget.

Guba, E. G., \& Lincoln, Y. S. (1989). Fourth generation evaluation. Newbury Park, CA: SAGE.

Heath, C., Hindmarsh, J., \& Luff, P. (2011). Video in qualitative research. London, UK: SAGE

Heath, C., Luff, P., \& Sanchez Svensson, M. (2007). Video and qualitative research: Analysing medical practice and interaction. Medical Education, 41, 109-116

Hjelle, K. M., Skutle, O., Førland, O., \& Alvsvåg, H. (2016). The reablement team’s voice: a qualitative study of how an integrated multidisciplinary team experiences participation in reablement. Journal of Multidisciplinary Healthcare, 9, 575-585 
Kakuma, R., Minas, H., van Ginneken, N., Dal Poz, M. R., Desiraju, K., Morris, J. E., . . . Scheffler, R. M. (2011). Human resources for mental health care: current situation and strategies for action. Lancet, 378, 1654-1663

Kinsella, E. A. (2010). The art of reflective practice in health and social care: Reflections on the legacy of Donald Schön. Reflective Practice, 11, 565-575

Korpi, H., Peltokallio, L., \& Piirainen, A. (2014). The story models of physiotherapy students’ professional development. Narrative research. The European Journal of Physiotherapy, 16, 219-229

Kvale, S., \& Brinkmann, S. (2009). Interviews: Learning the craft of qualitative research interviewing (2nd ed. ed.). Los Angeles, CA: SAGE.

Lähteenmäki, M. L. (2005). Reflectivity in supervised practice: Conventional and transformative approaches to physiotherapy. Learning in Health and Social Care, 4, 18-28. doi:10.1111/j.1473-6861.2005.00080.x

Lave, J., \& Wenger, E. (1991). Situated learning: Legitimate peripheral participation. Cambridge, UK: Cambridge University.

Legg, L., Gladman, J., Drummond, A., \& Davidson, A. (2016). A systematic review of the evidence on home care reablement services. Clinical Rehabilitation, 30, 741-749. doi:10.1177/0269215515603220

Lincoln, Y. S., \& Guba, E. G. (1985). Naturalistic inquiry (Vol. 75). Los Angeles, CA: SAGE.

Moe, A., \& Brataas, H. V. (2016). Interdisciplinary collaboration experiences in creating an everyday rehabilitation model: a pilot study. Journal of Multidisciplinary Healthcare, 9, 173-182S 
Ness, N. E., Laberg, T., Haneborg, M., Granbo, R., Færevaag, L., \& Butli, H. (2012). Hverdagsmestring og hverdagsrehabilitering [Everyday Coping and everyday rehabilitation].

Nicholls, D. A., \& Gibson, B. E. (2010). The body and physiotherapy. Physiotherapy Theory and Practice, 26, 497-509

Normann, B., Sørgaard, K. W., Salvesen, R., \& Moe, S. (2014). Clinical guidance of community physiotherapists regarding people with MS: Professional development and continuity of care. Physiotherapy Research International, 19, 25-33

Pettersson, C., \& Iwarsson, S. (2017). Evidence-based interventions involving occupational therapists are needed in re-ablement for older community-living people: A systematic review. British Journal of Occupational Therapy, 80, 273-285

QSR International. What is NVivo. Retrieved from http://www.qsrinternational.com/ Rabiee, P., \& Glendinning, C. (2011). Organisation and delivery of home care re-ablement: What makes a difference? Health \& Social Care in the Community, 19, 495-503

Randström, K. B., Wengler, Y., Asplund, K., Svedlund, M. (2012). Working with 'handsoff'support: a qualitative study of multidisciplinary teams' experiences of home rehabilitation for older people. International journal of older people nursing, 9, 25-33

Reeves, S., Lewin, S., Espin, S., \& Zwarenstein, M. (2010). Interprofessional teamwork for health and social care. London, UK: Blackwell-Wiley.

Reeves, S., Xyrichis, A., \& Zwarenstein, M. (2018). Teamwork, collaboration, coordination, and networking: why we need to distinguish between different types of interprofessional practice. Journal of Interprofessional Care, 32, 1-3

Schön, D. (1983). The reflective practitioner: How professionals think in action. New York, NY: Basic Books. 
Schön, D. (1991). The reflective practitioner: How professionals think in action. Farnham, UK: Ashgate Publishing.

Sørvoll, M., Obstfelder, A., Normann, B., \& Øberg, G. K. (2016). Management and dissemination of professional knowledge: Physiotherapists' perceptions of supervision of dedicated aides working with children with Cerebral Palsy (In Press) The University of Tromsø The Arcitic University of Norway

Stainsby, K., \& Bannigan, K. (2012). Reviewing work-based learning opportunities in the community for physiotherapy students: An action research study. Journal of Further and Higher Education, 36, 459-476. doi:10.1080/0309877X.2011.643769

Tessier, A., Beaulieu, M. D., McGinn, C. A., \& Latulippe, R. (2016). Effectiveness of reablement: A systematic review. Healthcare Policy, 11, 49-59

Thylefors, I., Persson O., Hellström D. (2005). Team types, perceived efficiency and team climate in Swedish cross-professional teamwork. Journal of Interprofessional Care, $19,102-114$

Wenger, E. (1998). Communities of practice: Learning, meaning, and identity. Cambridge, UK: Cambridge University Press.

World Health Organization. (2006). The world health report 2006: Working together for health. Retrieved from http://www.who.int/whr/2006/whr06_en.pdf?ua=1

World Medical Association. (2017). WMA declaration of Helsinki - ethical principles for medical research involving human subjects. Retrieved from https://www.wma.net/policies-post/wma-declaration-of-helsinki-ethical-principles-formedical-research-involving-human-subjects/ 